\title{
Ship Targets Discrimination Algorithm in SAR Images Based on Hu Moment Feature and Texture Feature
}

\author{
Lei LIU*, Yang ZHANG, Hai-Bo ZHANG, Qi FENG, Ting-Jun LI \\ Naval Aeronautical and Astronautical University, Yantai 264001, China \\ *e-mail: ythyliulei@sina.com
}

\begin{abstract}
To discriminate the ship targets in SAR images, this paper proposed the method based on combination of $\mathrm{Hu}$ moment feature and texture feature. Firstly,7 $\mathrm{Hu}$ moment features should be extracted, while gray level co-occurrence matrix is then used to extract the features of mean, variance, uniformity, energy, entropy, inertia moment, correlation and differences. Finally the k-neighbour classifier was used to analysis the 15 dimensional feature vectors. The experimental results show that the method of this paper has a good effect.
\end{abstract}

\section{Introduction}

Marine environmental monitoring has become the common concern for all countries in the world [1].Ships are the main tools to develop Marine, so how to effectively get the ship information is becoming a hot spot of current research. Automatic target recognition (ATR) system can extract information of ship targets in SAR image, mainly includes three phases: detection, discrimination and classification [2]. The detection phase can remove the region that can't be the target area, while discrimination phase can remove natural clutter and artificial clutter false alarm, the classification phase is to classify ship target recognition. Target discrimination is the intermediate link of the ATR system and the basis of ship target classification [3].

Feature extraction is the foundation of SAR images of ship target identification [4,5]. In the late 1980s, Burl and Novak firstly put forward by using texture feature for SAR image target discrimination in Lincoln laboratory, in the next 30 years, scholars from all over the world had made many achievements in the discrimination of feature extraction [6]. Hu invariant moments features can reflect the global features of the image because of rotation, scaling and translation invariance, but it can't descript the details of the image effectively, and the recognition rate was be influenced. Gray level co-occurrence matrix describes the image gray level of the internal texture structure through the study of spatial correlation characteristics, which has strong adaptability and robustness [7]. Combining with $7 \mathrm{Hu}$ invariant moments features and 8 texture feature extraction to form the feature vector $15 \mathrm{~d}$, and $\mathrm{k}$-neighbor classifier are classified, and separately adopt the $\mathrm{Hu}$ invariant moment features and texture features of the classification results are compared, the results show that this algorithm can effectively improve the accuracy of classification $[8,9]$.

\section{Feature Extraction of Discrimination}

\subsection{Hu Moment Feature Extraction}

Geometric moment was put forward by M.K.Hu in 1962, it provided theory basis for the choice of target torque characteristics of images because of translation, rotation and scaling invariance [10].

For the gray level distribution of $1 f(x, y)$, the $(p+q)$ moment is defined as:

$$
\begin{gathered}
m_{p q}=\iint x^{p} y^{q} f(x, y) d x d y \quad p, q=0,1,2, \cdots \\
(p+q) \text { central moment is defined as: } \\
\mu_{p q}=\iint\left(x-x_{0}\right)^{p}\left(y-y_{0}\right)^{q} f(x, y) d x d y
\end{gathered}
$$

The centroid $\left(x_{0}, y_{0}\right)$ is:

$$
x_{0}=\frac{m_{10}}{m_{00}}, \quad y_{0}=\frac{m_{01}}{m_{00}}
$$

In the discrete state, the $(p+q)$ ordinary moment and central moment of $f(x, y)$ is as follows:

$$
m_{p q}=\sum_{x=1}^{M} \sum_{y=1}^{N} x^{p} y^{q} f(x, y)
$$




$$
\mu_{p q}=\sum_{x=1}^{M} \sum_{y=1}^{N}\left(x-x_{0}\right)^{p}\left(y-y_{0}\right)^{q} f(x, y) \quad p, q=0,1,2, \cdots
$$

As the image changes, $m^{p q}$ also changes and $\mu^{p q}$ has translational invariance.

The normalized central moment is:

$$
y_{p q}=\frac{\mu_{p q}}{\mu_{00}{ }^{r}}, r=\frac{p+q+2}{2}, p+q=2,3, \cdots
$$

$\mathrm{Hu}$ constructed seven moment invariants by using the second order and third order central moments, they can keep unchanged translation, scaling and rotation under the condition of the continuous image, the definitions are:

$$
\begin{gathered}
I_{1}=y_{20}+y_{02} \\
I_{2}=\left(y_{20}-y_{02}\right)^{2}+4 y_{11}^{2} \\
I_{3}=\left(y_{30}-3 y_{12}\right)^{2}+\left(3 y_{21}-y_{03}\right)^{2} \\
I_{4}=\left(y_{30}+y_{12}\right)^{2}+\left(y_{21}+y_{03}\right)^{2} \\
I_{5}=\left(y_{30}-3 y_{21}\right)\left(y_{21}+y_{03}\right)\left[\left(y_{30}+y_{21}\right)^{2}-3\left(y_{21}+y_{03}\right)^{2}\right] \\
+\left(3 y_{21}-y_{03}\right)\left(y_{21}+y_{03}\right)\left[3\left(y_{30}+y_{12}\right)^{2}-\left(y_{21}+y_{03}\right)^{2}\right] \\
(11) \\
I_{6}=\left(y_{20}-y_{02}\right)\left[\left(y_{30}+y_{12}\right)^{2}-\left(y_{21}+y_{03}\right)^{2}\right] \\
+4 y_{11}\left(y_{30}+y_{12}\right)\left(y_{21}+y_{03}\right) \\
I_{7}=\left(3 y_{21}-3 y_{03}\right)\left(y_{30}+y_{12}\right)\left[\left(y_{30}+y_{12}\right)^{2}-3\left(y_{21}+y_{03}\right)^{2}\right] \\
+\left(y_{30}-3 y_{12}\right)\left(y_{21}+y_{03}\right)\left[3\left(y_{30}+y_{12}\right)^{2}-\left(y_{21}+y_{03}\right)^{2}\right]
\end{gathered}
$$

So the target subspace is $V=\left[I_{1}, I_{2}, I_{3}, I_{4}, I_{5}, I_{6}, I_{7},\right]$.

\subsection{Texture Feature Extraction based on Gray Level Co-occurrence Matrix}

SAR image contains abundant texture information. Texture feature extraction method based on gray level co-occurrence matrix is a classic method which can describe the randomness and texture details well and it has the characteristics of strong adaptability [11].

As shown in figure 1, gray level co-occurrence matrix calculated the probability of pixel $(i, j)$ at a distance of $d$ on the direction of $\theta$ :

$$
p=p_{i j d \theta}(i, j=0,1,2, \cdots, l-1)
$$

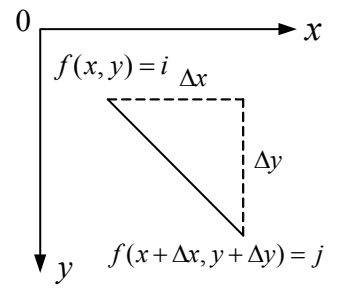

Figure 1 Gray Level Co-Occurrence Matrix Principle Diagram

$p_{i j d \theta}$ is the number of pixels to appear which gray is $i$ and $j$ ata distance of $d$ on the direction of $\theta ; l$ is the image gray-scale series.

The angle $\theta$ can do the following provisions:

$$
\theta=\left\{\begin{array}{l}
0^{0}, \Delta x= \pm 1, \Delta y=0 \\
45^{0}, \Delta x=1, \Delta y= \pm 1 \\
90^{\circ}, \Delta x=0, \Delta y= \pm 1 \\
135^{0}, \Delta x=-1, \Delta y= \pm 1
\end{array}\right.
$$

Although gray level co-occurrence matrix can reveal the texture rule of images, but it cannot directly be a digital feature of image classification. Therefore it is necessary to define some obvious physical meaning statistics based on gray level co-occurrence matrix that can describe the thickness and direction of the texture. This paper extracted eight texture characteristics that are mean, variance, uniformity, energy, entropy, inertia moment, correlation and differences [12].

(1) Mean

$$
M=1 / m n \sum_{i=0}^{m} \sum_{j=0}^{n} p_{i j d \theta}
$$

$m, n$ are rows and columns of the gray level co-occurrence matrix

(2) Variance

$$
V=\sum_{i=0}^{m} \sum_{j=0}^{n}(i-\mu)^{2} p_{i j d \theta}^{*}
$$

$\mu=\sum_{i=0}^{m} \sum_{j=0}^{n} i p_{i j d \theta}^{2}, p_{i j d \theta}^{*}$ is the normalized value of $p_{i j d \theta}$.

(3) Uniformity

$$
H=\sum_{i=0}^{m} \sum_{j=0}^{n} \frac{p_{i j d \theta}^{*}}{1+(i-j)^{2}}
$$

Uniformity can measure local homogeneity, if the local uniform, the value is larger; otherwise, the value is smaller.

(4) Energy

$$
A_{S M}=\sum_{i=0}^{m} \sum_{j=0}^{n} p_{i j d \theta}^{2}
$$

Energy also calls the angular second moment, it reflects the image grayscale distribution uniformity. 
When angular second moment is large and texture is rough, energy is large; conversely, texture is thin, energy is small.

(5) Entropy

$$
E=-\sum_{i=0}^{m} \sum_{j=0}^{n} p^{*}(i, j) \lg p_{i j d \theta}^{*}
$$

Entropy is associated with the density of texture. If the image is full of fine texture, the entropy is largest; if the texture is less, the entropy is smaller.

(6) Inertia moment

$$
C_{1}=\sum_{i=0}^{m} \sum_{j=0}^{n}(i-j)^{2} p_{i j d \theta}^{*}
$$

This parameter represents the distance of the larger elements from the extent of the principal diagonal, if texture is rough, the inertia moment is small, and otherwise, the inertia moment is large.

(7) Correlation

$$
\begin{array}{r}
C_{2}=\sum_{i=0}^{m} \sum_{j=0}^{n} \frac{(i-\mu)(j-\mu) p_{i j d \theta}^{*}}{\sigma^{2}} \\
\text { with } \sigma^{2}=\sum_{i=0}^{m} \sum_{j=0}^{n}(i-\mu)^{2} p_{i j d \theta}
\end{array}
$$

Correlation is used to measure the similarity degree of gray level co-occurrence matrix elements on the row or column direction. When the matrix element values are equal, the value is big; On the contrary, the value is small.

(8) Differences

$$
D=\sum_{i=0}^{m} \sum_{j=0}^{n}|i-j| p_{i j d \theta}^{*}
$$

Differences can describe the difference of gray scale image pixels, the greater the difference, the easier to identify.

\section{Classification Method}

Classification algorithm flow chart is shown in figure 2.

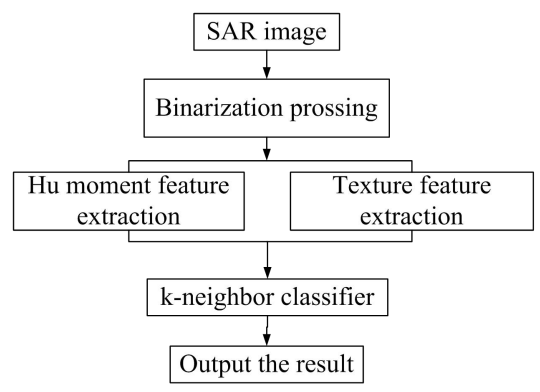

Figure 2 Algorithm Process
$\mathrm{K}$-neighbor classifier is a mature method in theory. The idea of the method is: to a sample in the space, if most of the sample in the $\mathrm{k}$ most similar sample belongs to a category, then the sample also belongs to this category.

First the sample of the SAR image should be preprocessed, while the binary images would be got. Then we extract the seven invariant moment feature and eight texture features and get a set of 15 dimensional feature vectors. Therefore every image can be represented by the feature vector of the $15 \mathrm{~d}$ and we would get an $\mathrm{n} \times 15$ dimensional feature vector database. Lastly we will put the database into k-neighbor classifier and calculate the classification accuracy.

\section{Result of the Experiment and Analysis}

The experimental data is taken from the $\mathrm{C}$ band RADARSAT-2 satellite. The resolution of image is $3 \mathrm{~m}$. Ship target and clutter images are cut into 60 samples of $100 \times 100$ pixel size, Put the image samples into morphology filter and get the binary image.
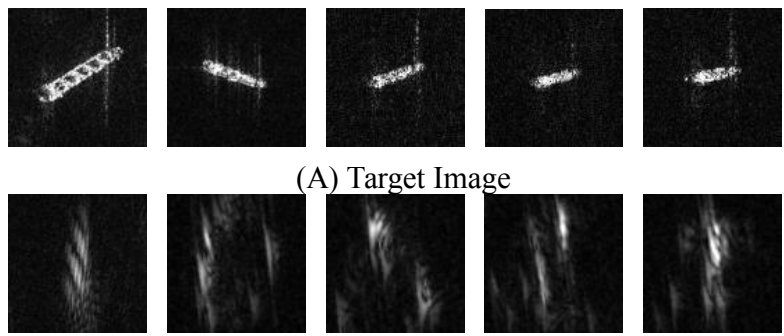

(A) Target Image
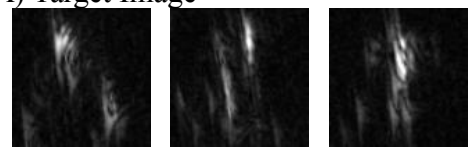

(B) Clutter Image

Figure 3 Image Samples of Thesar Image
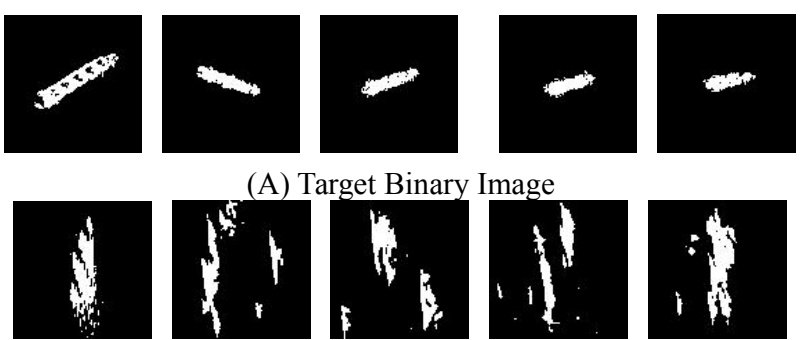

(B) Clutter Binary Image

Figure 4 Target And Clutter Binary Image

This section selected 60 target samples and 60 clutter samples. $50 \%$ of the samples are as the training sample and $50 \%$ as the test samples. According to the $\mathrm{Hu}$ invariant moment features and gray level co-occurrence we get the $60 \times 15$ dtarget sample data and $60 \times 15 \mathrm{~d}$ clutter sample data. Put the data into k-neighbor classifier for training and testing to get the identification results, and then separate with the results of the $\mathrm{Hu}$ invariant moment features and texture features. In this paper, we set $\mathrm{k}=3$. 
Table 1 Classification Results

\begin{tabular}{|l|l|l|l|}
\hline \multicolumn{1}{|c|}{ classification } & Hu moment feature & Texture feature & Feature of this paper \\
\hline $\begin{array}{l}\text { True number of } \\
\text { target classification }\end{array}$ & 26 & 29 & 30 \\
\hline $\begin{array}{l}\text { False number of } \\
\text { target classification }\end{array}$ & 4 & 1 & 0 \\
\hline $\begin{array}{l}\text { True number of } \\
\text { clutter classification }\end{array}$ & 25 & 28 & 29 \\
\hline $\begin{array}{l}\text { False number of } \\
\text { clutter classification }\end{array}$ & 5 & 2 & 1 \\
\hline $\begin{array}{l}\text { True number of } \\
\text { clutter classification }\end{array}$ & 51 & 57 & 59 \\
\hline $\begin{array}{l}\text { False number of } \\
\text { clutter classification }\end{array}$ & 9 & 3 & 1 \\
\hline Accuracy & $85 \%$ & $95 \%$ & $98.3 \%$ \\
\hline
\end{tabular}

By comparing test results above, we can get that the classification accuracy of using $\mathrm{Hu}$ moment feature alone to discriminate is $85 \%$, because although $\mathrm{Hu}$ moment can reflect the global features of the image, it cannot describe the detail of the image well. So that caused the image description is not complete and classification accuracy is relatively low. Gray level co-occurrence matrix was used to extract texture structure, which has strong adaptability and robustness. Its classification effect is better; Combining $\mathrm{Hu}$ moment features and texture features can describe the image completely and the classification effect is remarkable.

\section{Conclusion}

This paper proposed a ship targets discrimination algorithm in SAR images based on $\mathrm{Hu}$ moment feature and texture feature. $\mathrm{Hu}$ moment feature reflects the shape information of the image and texture reflects all sorts of objective information of the image. Combining the $\mathrm{Hu}$ invariant moment features and the texture features extracted by gray level co-occurrence matrix, then put the data into k-neighbor classifier to discriminate. The experimental results show that this algorithm can effectively improve the classification accuracy and classification effect.

\section{References}

Here are some examples:

1. M. Liao, C. Wang, Y. Wang, and L. Jiang, "Using SAR images to detect ships from sea clutter," IEEE Geosci. Remote Sens. Lett., vol. 5, no. 2,pp. 194-198, Apr. 2008

2. M. C. Burl, G. J. Owirka, and L. M. Novak, "Texture discrimination in synthetic aperture radar imagery," in Proc. 23rd Asilomar Conf. Signals,
Syst., Compute., 1989, pp. 399-404.

3. G. Gao, "An improved scheme for target discrimination in high-resolution SAR images," IEEE Trans. Geosci. Remote Sens., vol. 49, no. 1, pp. 277-294, Jan. 2011

4. O. Tuzel, F. Porikli, and P. Meer, "Region covariance: A fast descriptor for detection and classification," in Proc. ECCV, 2006, pp. 589-600

5. D. E. Kreithen, S. D. Halversen, and G. J. Owirka, "Discriminating targets from clutter," Lincoln Lab. J., vol. 6, no. 1, pp. 25-52, 1993

6. C. Zhu, H. Zhou, R. Wang, and J. Guo, "A novel hierarchical method of ship detection from space borne optical image based on shape and texture features," IEEE Trans. Geosci. Remote Sens., vol. 48, no. 9, pp. 3446-3456, Sep. 2010

7. D. Pastina, F. Fico, and P. Lombardo, "Detection of ship targets in COSMO-Sky Med SAR images," in Proc. IEEE RADAR, 2011,pp. 928-933

8. S. Brusch, S. Lehner, T. Fritz, M. Soccorsi, A. Soloviev, and B. van Schie, "Ship surveillance with Terra SAR-X," IEEE Trans. Geosci. Remote Sens., vol. 49, no. 3, pp. 1092-1103, Mar. 2011.

9. J. Chai, "Study of rejection algorithm and feature extraction technique on radar HRRP target recognition," Ph.D. Thesis, Xidian Univ., Xi'an, China, 2010

10. S. I. Hwang and K. Ouchi, "On a novel approach using MLCC and CFAR for the improvement of ship detection by synthetic aperture radar," IEEE Geosci. Remote Sens. Lett., vol. 7, no. 2, pp. 391-395, Apr. 2010.

11. M. Liao, C. Wang, Y. Wang, and L. Jiang, "Using SAR images to detect ships from sea clutter," IEEE Geosci. Remote Sens. Lett., vol. 5, no. 2,pp. 194-198, Apr. 2008.

12. D. J. Crisp, The State-of-the-Art in Ship Detection in Synthetic Aperture Radar Imagery. Australia: DSTO Inf. Sci. Lab., 2004. 